This is the accepted version of the article:

Cabana, L.; Ballesteros, B.; Batista, E.; Magén, C.; Arenal, R.; Orõ-Solé, J.; Rurali, R.; Tobias, G.. Synthesis of Pbl 2 single-layered inorganic nanotubes encapsulated within carbon nanotubes. Advanced Materials, (2014). 26. 13: 2016 - . 10.1002/adma.201305169.

Available at: https://dx.doi.org/10.1002/adma.201305169 


\section{Synthesis of $\mathbf{P b I}_{2}$ Single-Layered Inorganic Nanotubes Encapsulated Within Carbon Nanotubes}

To the memory of Prof. John D. Corbett, Distinguished Professor at Iowa State University.

Laura Cabana, Belén Ballesteros, Eudar Batista, César Magén, Raúl Arenal, Judith Oro-Solé, Riccardo Rurali, and Gerard Tobias*

L. Cabana, E. Batista, Dr. J. Oro-Solé, Dr. R. Rurali, Dr. G. Tobias

Institut de Ciència de Materials de Barcelona (ICMAB-CSIC), Campus UAB, 08193

Bellaterra, Barcelona, Spain

E-mail: gerard.tobias@icmab.es

Dr. B. Ballesteros

ICN2 - Institut Catala de Nanociencia i Nanotecnologia, Campus UAB, 08193 Bellaterra, Barcelona, Spain

Dr. C. Magén, Dr. R. Arenal

Laboratorio de Microscopías Avanzadas (LMA), Instituto de Nanociencia de Aragón (INA), Universidad de Zaragoza, 50018 Zaragoza, Spain

ARAID Foundation, 50018 Zaragoza, Spain

Dr. C. Magén

Departamento de Física de la Materia Condensada, Universidad de Zaragoza, 50009 Zaragoza, Spain

Keywords: filled carbon nanotubes, metal halide, single-walled inorganic nanotubes, DFT calculations, core-shell template assisted growth

The isolation and growth of single layers of a wide variety of inorganic materials is of interest for both fundamental research and advanced applications. ${ }^{[1]}$ When an individual layer is seamlessly wrapped into a cylinder, the resulting single-layered nanotube combines the characteristics of both two-dimensional (2D) and one-dimensional (1D) materials. ${ }^{[2]}$ Yet, despite their interest, reports on single-walled nanotubes are scarce because their multiwalled counter parts are in general favoured during growth. ${ }^{[3]}$ Here we report on a versatile approach that allows the formation of high-quality, single-crystalline single-layered inorganic nanotubes. To achieve this, we employed a solvent-free high temperature route using the inner cavities of carbon nanotubes as a hosting templates. Lead iodide single-layered nanotubes, ranging from 3.5 to $7.5 \mathrm{~nm}$ in diameter, were prepared by this methodology. Using aberrationcorrected electron microscopy and first-principles quantum mechanical calculations we have 
explored the fundamental physics and chemistry of the resulting materials. Remarkably, our theoretical studies show that the single-layered nanotubes are stable without the presence of the sheathing carbon and their electronic structure is found to be diameter independent. The method presented here offers wide versatility towards the formation of tubular single-layered nanomaterials of different nature whose diameter is merely determined by the host.

Carbon nanotubes (CNTs) are a highly versatile platform for the template assisted growth of nanomaterials, given that both their internal space and the outer surface allow the formation of tailored nanostructures. ${ }^{[4]}$ The encapsulation of materials inside CNTs results in the formation of extremely narrow $1 \mathrm{D}$ nanowires ${ }^{[5]}$ that can go down to a chain of single atoms forming an "atomic necklace”, ${ }^{6]}$ The use of filled carbon nanotubes has been recently explored in radiology, ${ }^{[7]}$ nanothermometry, ${ }^{[8]}$ molecular electronics, ${ }^{[9]}$ water confinement, ${ }^{[10]}$ cell tracking ${ }^{[11]}$ and as nanoscale reaction vessels for the controlled growth of graphene nanoribbons. ${ }^{[12]}$ However, in spite of the versatility that the nanoencapsulation into CNTs offers, the use of the inner cavities for the growth of single-layered inorganic materials has not been realized to date. In this report, we present the template-assisted growth of singlelayered lead iodide crystals. Lead iodide is a semiconducting material with interest for its electroluminescence, photoluminiscence and non-linear optical effects. ${ }^{[13]}$ It is a good candidate for X-ray and $\gamma$-ray detectors, thin film transistors and more recently organo-lead iodide perovskites are employed in efficient hybrid solar cells. ${ }^{[14]}$ Multiwalled nanotubes and nanoclusters of lead iodide have recently been prepared by template assisted growth using $\mathrm{WS}_{2}$ nanotubes for the former ${ }^{[15]}$ and supramolecular cages for the latter, ${ }^{[16]}$ but their singlewalled counterparts have not been observed. We reasoned that this could be achieved by using the cavities of multiwalled carbon nanotubes (MWCNTs) due to their narrower cavity compared to that of $\mathrm{WS}_{2}$.

The tubular single-layers of $\mathrm{PbI}_{2}$ were prepared by molten phase capillary wetting ${ }^{[5]}$ using steam purified and open-ended CNTs. ${ }^{[17]}$ The successful growth of single-layered 
nanotubes within the CNT walls was confirmed by aberration-corrected high-resolution transmission electron microscopy (HRTEM) and scanning transmission electron microscopy (STEM) at $80 \mathrm{kV}$. First-principles density functional theoretical calculations allowed the determination of the stability and electronic properties of the prepared materials.

The structure of the single-layered $\mathrm{PbI}_{2}$ nanotubes was initially analyzed by HRTEM. In Figure 1a, a single-layered nanotube with an outermost external diameter of $3.94 \mathrm{~nm}$ is observed (area A). Taking into account that each layer of $\mathrm{PbI}_{2}$ is actually composed by a I-PbI repeating unit in which a layer of $\mathrm{Pb}$ atoms is sandwiched between two layers of I atoms (see schematic representation), an external diameter of $3.94 \mathrm{~nm}$ actually corresponds to a nanotube of about $3.5 \mathrm{~nm}$ (taking $\mathrm{Pb}$ as the central point). We will therefore refer to the diameter of the $\mathrm{PbI}_{2}$ nanotubes to this central $\mathrm{Pb}$ layer. It is worth noticing the ease in which the tubular structure of $\mathrm{PbI}_{2}$ accommodates in the core of the host, overcoming for instance a narrowing of the CNT (area B) or even a dramatic 30\% increase in diameter from $3.5 \mathrm{~nm}$ to $4.5 \mathrm{~nm}$ (area C). A high magnification image of the tip of the $\mathrm{PbI}_{2}$ nanotube is presented in Figure 1b, where its high degree of crystallinity can be better appreciated. Image simulation along with the employed model structure is also included for comparison. Since the chirality of each of the layers of the carbon nanotube is unknown, all the CNTs were taken as zig-zag. Despite small differences, the simulated image is indeed in good agreement with the presence of a lead iodide single-layered nanotube. The distinctive curvature of the prepared inorganic nanotubes can also be directly appreciated by both HRTEM (Figure 1c; average diameter 4.38 nm) and by high-angle annular dark field (HAADF) imaging in high-resolution STEM (see Supporting Information Figure S1). HAADF imaging provides Z-contrast images which scale with a power of the atomic number, thus facilitating the detection of heavy elements confined within carbon nanotubes or attached to their external walls down to the atomic scale. ${ }^{[18]}$ Therefore in HAADF STEM a large contrast is observed between $\mathrm{PbI}_{2}$ and $\mathrm{C}$ from the host. The wall thickness measured in the HAADF STEM image is about $0.4 \mathrm{~nm}$ (Figure S1), 
consistent with that of a single-layer of $\mathrm{PbI}_{2}$. Bulk $\mathrm{PbI}_{2}$ has an interlayer spacing of $0.6986 \mathrm{~nm}$ which takes into account the van der Waals spacing, ${ }^{[19]}$ therefore a smaller thickness is expected when measuring an individual single layer. In order to unambiguously determine the successful formation of the tubular structures, HAADF imaging in STEM and x-rays energy dispersive spectroscopy (EDS) experiments were combined to produce elemental profiles of a region of the sample presenting a white tubular contrast (Figure 1d). As expected, a higher intensity of both constituent elements, I and $\mathrm{Pb}$, is detected at the edges compared to the central space confirming the tubular nature of the grown material. For comparison we also analyzed a nanorod of $\mathrm{PbI}_{2}$ within the CNT host, where the highest intensity for both elements is encountered at the center of the profile, at variance with the hollow structures discussed thus far (see Supporting Information, Figure S2).

Encouraged by the successful formation of $\mathrm{PbI}_{2}$ nanotubes, we further investigated the role that the diameter of the confining cage has on the formation of the inorganic nanocrystals. For that purpose, a sample of open-ended CNTs with inner diameters ranging from $1.5 \mathrm{~nm}$ to $9 \mathrm{~nm}$ was employed, having a larger proportion of nanotubes between 4 and $6 \mathrm{~nm}$. We measured the diameter of the inner cavity of the CNT host when either a nanorod or a singlelayered nanotube was present and the resulting histograms are plotted in Figure 2a. Interestingly, despite the confined growth of nanorods can take place irrespective of the diameter of the host, the formation of nanotubes preserve a threshold at $4 \mathrm{~nm}$. A large fraction of $\mathrm{PbI}_{2}$ nanotubes are present within CNTs with diameters of about 4.5-5 nm merely reflecting the diameter distribution of the host in the starting material. Taking into account that the grown $\mathrm{PbI}_{2}$ nanotubes are about $0.5 \mathrm{~nm}$ narrower than the host, single-layered $\mathrm{PbI}_{2}$ nanotubes from 3.5 to $7.5 \mathrm{~nm}$ in diameter can be successfully prepared with this approach. Therefore one can envisage that tailored $\mathrm{PbI}_{2}$ nanotubes with regular diameters are indeed possible to prepare by using a hosting template with uniform diameters. 
To understand the role of the tubular template, we have performed first-principles electronic structure calculations of $\mathrm{PbI}_{2}$ nanotubes with diameters ranging from 3 to $5 \mathrm{~nm}$. The metal halide nanotubes were modeled by rolling up a fragment (of variable size) of an individual layer of bulk $\mathrm{PbI}_{2 .}{ }^{[19]}$ Initially we have calculated the wrapping energy, the energy required to roll up a flat monolayer into a tube (Figure 2b). The energy cost of the wrapping is rather moderate (it is larger than $1 \mathrm{eV}$ per nanotube unit cell only for the narrower systems investigated) and, as expected, it decreases as the diameter of the nanotube increases. This energy is always largely exceeded by the encapsulation energy of the $\mathrm{PbI} 2$ nanotubes, the van der Waals $\mathrm{PbI}_{2}-\mathrm{C}$ interlayer interaction that we found to be approximately $2.75 \mathrm{eV} \mathrm{nm}^{-2}$ (we obtain a very similar value for the interlayer interaction in graphite), thus easing the formation of the nanotubes. This difference decreases at smaller diameters and makes the synthesis of narrow tubes less likely, favoring the higher relative stability of $\mathrm{PbI} 2$ nanorods in such a size range. The theoretical calculations also predict stability for the single-layered $\mathrm{PbI}_{2}$ nanotubes in case the template was removed: all the stand-alone nanotubes considered turned out to be stable. All the tubes studied are wide band-gap semiconductors, with an energy band-gap very similar to the one of bulk $\mathrm{PbI}_{2}$ and scarcely dependent on the tube diameter or wrapping axis (see Figure 2c), as previously observed for BN nanotubes. ${ }^{[20]}$ All the calculated band-gaps are within $0.056 \mathrm{eV}$, a range further reduced to $0.09 \mathrm{eV}$ if the two narrowest, and at present, unrealistic diameters are discarded.

The possibility of growing single-layered nanotubes using nanotubular templates in such a range of diameters ( 3.5 to $7.5 \mathrm{~nm}$ ) is remarkable. Figure $2 \mathrm{~d}$ summarizes the $\mathrm{PbI}_{2}$ nanostructures that have been prepared so far using their template-assisted growth. Within the 0-dimensional range, nanoclusters of $\mathrm{PbI}_{2}$ (with molecular formula $\mathrm{Pb}_{10} \mathrm{I}_{20}$ ) have recently been prepared employing supramolecular cages. ${ }^{[16]}$ More examples are available with 1dimensional nanostructures. When employing either single-walled or double-walled carbon nanotubes (1-4 nm in diameter), $\mathrm{PbI}_{2}$ nanorods, and not nanotubes, are always observed. ${ }^{[21]}$ 
The use of inorganic WS 2 nanotubes (inner and outer diameters of about $10 \mathrm{~nm}$ and $20 \mathrm{~nm}$ respectively) as hosts allows the formation of multiwalled $\mathrm{PbI}_{2}$ nanotubes (3-5 layers) as well as $\mathrm{PbI}_{2}$ inorganic nanorods. ${ }^{[15]}$ Model calculations indicate that $\mathrm{PbI}_{2}$ multiwalled nanotubes become stable inside $\mathrm{MoS}_{2}$ (model for $\mathrm{WS}_{2}$ ) only when the diameter of the host is larger than $12 \mathrm{~nm}$; below this diameter the $\mathrm{PbI}_{2}$ crystallizes as a nanorod. ${ }^{[22]}$ This is in agreement with the experimental observations. ${ }^{[22]}$ Other metal halides, including $\mathrm{BiI}_{3}$ and $\mathrm{SbI}_{3}$ have also been confined within the $\mathrm{WS}_{2}$ nanocapillaries leading also to the formation of multiwalled nanotubes and nanorods. It is only when carbon nanotubes are employed, that single-layered $\mathrm{PbI}_{2}$ can be prepared. The filling of $\mathrm{PbI}_{2}$ occurs in a fast and effective manner and can be achieved after annealing the bulk metal halide in the presence of carbon nanotubes in only 10 minutes. Higher encapsulation efficiencies are obtained after annealing for longer periods of time. The formation of single-layered $\mathrm{PbI}_{2}$ tubular structures is also accompanied by the presence of $\mathrm{PbI}_{2}$ nanorods, and several nanotube-nanorod junctions are present. Molecular dynamic simulations suggested that $\mathrm{PbI}_{2}$ initially fills as a molten drop into tubular templates; this can then lead to the spread of melt over the inner surface of the host with crystallization of $\mathrm{PbI}_{2}$ nanotubes on cooling (into chalcogenide hosts). ${ }^{[23]}$ The diameter of the CNT models employed for the simulations lie within the diameters encountered with single- and doublewalled CNTs. In this range of diameters, only the formation of tubular structures of one-atom in thickness (graphene-like) has been described by dynamic and static simulations. ${ }^{[24]}$ Therefore molecular dynamic studies with CNTs of larger diameter, such as those used in the present study, are needed to get further insights in these systems.

A great deal of interest is devoted towards the study of physical transformations that materials undergo in a confined nanoscale geometry. ${ }^{[25,26]}$ Electron microscopy has proven to be a powerful tool to directly monitor these changes in real time. ${ }^{[27]}$ Therefore, we next studied the dynamic effect of electron beam irradiation on several nanotube-nanorod junctions. Figure 3 presents a sequence of images of a $\mathrm{PbI}_{2}$ nanorod confined within an envolving $\mathrm{PbI}_{2}$ 
nanotube. Upon irradiation by the electron beam at $80 \mathrm{kV}$ (to reduce knock-on damage), a continuous blending of the nanorod onto the nanotube takes place until its complete dissolution, thus resulting in the formation of a continuous $\mathrm{PbI}_{2}$ nanotube. The conversion process has been observed in other nanotube-nanorod-nanotube junctions. The inverse phenomenon has also been observed, where a fragment of nanotube confined within two nanorods is "filled" resulting in a continuous nanorod (Supporting Figure S3). In both cases, the transformation from one type of nanostructure to the other takes place due to the irradiation by the electron beam. STEM analysis reveals that $\mathrm{PbI} 2$ nanorods can be composed of a narrower nanorod confined within a $\mathrm{PbI} 2$ single-layered nanotube (Supporting Figure S4). In this scenario, upon beam irradiation, the inner nanorod could be displaced within the $\mathrm{PbI}_{2}$ filling an empty void, as seems to be the case of the in-situ study presented in Figure S3. The opposite mechanism were the inner nanorod breaks apart leaving the shielding inorganic tube visible could also be considered but the nanotube to nanorod transformation observed in Figure 3 does not seem to fall in this scenario. In this sequence of images rather an atomic migration from the nanorod to the nanotubes seems to occur. To allow the accommodation of additional lead and iodine atoms into the tubular structure an atomic migration within the top/bottom part of the nanotubes would be necessary. The motion of confined materials, including metal halides, within the cavities of CNTs has been widely investigated. The displacement can be stimulated by either electron beam irradiation, like in the present study, or driven for instance by heat or electrical currents. ${ }^{[26,28,29]} \mathrm{A}$ wide variety of metal halides have been encapsulated within CNTs being electron microscopy the most commonly used technique for their investigation. ${ }^{[5,21,30]}$ In some cases electron beam irradiation has been reported to lead to chemical changes, typically after prolonged irradiation or under high acceleration voltages. ${ }^{[29,31]}$ Therefore the use of low voltage is desired when investigating these systems under the electron beam. The mechanism of transformation from nanotubes to 
nanorods and viceversa needs further investigation and even chemical changes cannot be disregarded at this stage.

As discussed, all the $\mathrm{PbI} 2$ nanotubes studied are wide band-gap semiconductors. Projections of the density of states (DOS) on the different chemical species reveal a peculiar localization of the band-edge states of different layers, for instance similarly to what have been reported for SiGe heterostructures. ${ }^{[32]}$ As can be seen in Figure 4a, valence states are almost exclusively made of I orbitals, while conduction states are mostly made of $\mathrm{Pb}$ orbitals. This is also evident in the real space representation of the wave-function of the higher (valence band) and lower (conduction band) states shown in Figure 4b. This behaviour can be in principle exploited in all those application where an efficient charge separation is required, such as solar cells where the efficiency of recombination of a created electron-hole pair must be minimized. The contribution of outer and inner I atoms is different, but of course this difference vanishes as the diameter increases and the curvature drops. Additionally, we observe that the conduction states exhibit a larger dispersion than the rather flat valence band (Figure 4c), thus electrons are expected to be more efficient charge carriers than holes in these tubular systems.

In conclusion, a solvent-free high temperature route has been explored that allows the formation of high-quality, single-crystalline inorganic nanotubes. We have produced an interesting hybrid core-shell structure combining two different tubular materials: singlelayered $\mathrm{PbI}_{2}$ nanotubes@CNTs. Above the threshold of $3.5 \mathrm{~nm}$, the single-layers of $\mathrm{PbI}_{2}$ can be easily moulded to the inner diameter of the hosting CNT template. The diameter of the inorganic nanotubes prepared herein is merely dependent on the diameter of the host. Therefore inorganic nanotubes of a given diameter can be prepared by simply using a sample of CNTs with uniform inner diameter. These coupled 1D nanostructures will offer very promising technological applications where materials having well defined electronic, optic or 
optoelectronic properties are required. The method is highly versatile opening up new horizons in the preparation of single-layered nanostructures of a wide variety of materials.

\section{Experimental Section}

Growth of $\mathrm{PbI}_{2}$ single-layered nanotubes: Chemical vapour deposition multiwalled carbon nanotubes (Thomas Swan Co.Ltd.) were steam treated in order to open their ends and purify the as-received material. ${ }^{[17]}$ Next the sample was treated with $\mathrm{HCl}$ to remove the now exposed catalytic metal nanoparticles. ${ }^{[33]}$ In an argon-filled glove box, purified CNTs (6 mg) and $\mathrm{PbI}_{2}(140 \mathrm{mg})$ were ground with an agate mortar and pestle until the mixture presented a uniform color. The mixture was transferred into a silica ampoule, evacuated and sealed under vacuum. The ampoule was placed into a furnace where it dwelled at $500{ }^{\circ} \mathrm{C}$ (above the melting point of lead iodide; mp $408{ }^{\circ} \mathrm{C}$ ); for different periods of time (from $10 \mathrm{~min}$ up to 86 h). Finally, the samples were cooled at room temperature.

HRTEM and STEM studies: TEM characterisation of the samples was performed on a JEOL JEM 1210 operated at $120 \mathrm{kV}$. HRTEM images were acquired in an image corrected FEI Titan Cube 60-300 microscope. STEM imaging and EDS chemical analyses were carried out in a probe corrected FEI Titan 60-300 microscope and a FEI Tecnai G2 F20 microscope. Both FEI Titan instruments were operated at $80 \mathrm{kV}$ with a point resolution $0.14 \mathrm{~nm}$. Samples for TEM characterisation were prepared by sonication of a small amount of powder in ethanol absolute. The dispersions were placed dropwise onto lacey carbon grids.

First-principles calculations: First-principles electronic structure calculations were performed within density-functional theory, as implemented in the SIESTA and VASP packages, ${ }^{[34]}$ expanding the one-electron wave function using a double- $\zeta$ basis set of numerical atomic orbitals and a plane-wave cutoff of $400 \mathrm{eV}$, respectively. We used the Local Density Approximation (LDA) for the exchange-correlation energy. ${ }^{[35]}$ The Brillouin zone was sampled with a grid of 12 points along the $\mathbf{k}_{\mathrm{z}}$ (z being the nanotube axis). The interlayer 
interaction between a $\mathrm{C}$ and a $\mathrm{PbI}_{2}$ nanotube was approximated with the graphene-PbI2 monolayer interaction energy (flat geometry) within the semi-empirical dispersion correction of Grimme. ${ }^{[36]}$ Due to the intrinsic incommensurability of graphene and lead iodide, we constructed the hybrid system by placing a $4 \times 4$ supercell of the flat $\mathrm{PbI}_{2}$ monolayer on top of a $7 \times 7$ supercell of graphene, with a compressive strain of $0.88 \%$ on the latter. The atomic positions were relaxed until all the forces were lower than $0.04 \mathrm{eV}^{-1}$ and the lattice parameter optimized in all the structures discussed.

\section{Supporting Information}

Supporting Information is available online from the Wiley Online Library or from the author.

\section{Acknowledgements}

This work was supported by MINECO (MAT2011-24757, FIS2012-37549-C05-05), EU FP7ITN RADDEL (290023) and Nanoaracat. The authors are grateful to Thomas Swan Co. Ltd. for supplying the MWCNT samples. L.C. acknowledges a JAE-Predoc fellowship (CSIC) and E.B. the CSIC-CITMA training and mobility program. L.C. is enrolled in the UAB PhD program. Aberration-corrected electron microscopy studies were conducted in the Laboratorio de Microscopias Avanzadas (LMA) at the Instituto de Nanociencia de Aragon (INA) - Universidad de Zaragoza (Spain).

Received: ((will be filled in by the editorial staff))

Revised: ((will be filled in by the editorial staff)) Published online: ((will be filled in by the editorial staff)) 3549.

[2] J. Goldberger, R. Fan, P. Yang, Acc. Chem. Res. 2006, 39, 239.

[3] C. N. R. Rao, A. Govindaraj, Adv. Mater. 2009, 21, 4208.

[4] D. Eder, Chem. Rev. 2010, 110, 1348. 
[5] R. R. Meyer, J. Sloan, R. E. Dunin Borkowski, A. I. Kirkland, M. C. Novotny, Science 2000, 289, 1324.

[6] R. Kitaura, R. Nakanishi, T. Saito, H. Yoshikawa, K. Awaga, H. Shinohara, Angew. Chem. Int. Ed. 2009, 48, 8298.

[7] S. Hong, G. Tobias, K. Al Jamal, B. Ballesteros, H. Ali Boucetta, S. Lozano Perez, P. Nellist, R. Sim, C. Finucane, S. Mather, K. Kostarelos, B. Davis, Nature Mater. 2010, 9, 485. [8] J. Y. Chen, A. Kutana, C. P. Collier, K. P. Giapis, Science 2005, 310, 1480.

[9] M. del Carmen Giménez-López, F. Moro, A. La Torre, C. J. Gómez-García, P. D. Brown, J. van Slageren, A. N. Khlobystov, Nature Commun. 2011, 2, 407.

[10] Y. Maniwa, K. Matsuda, H. Kyakuno, S. Ogasawara, T. Hibi, H. Kadowaki, S. Suzuki, Y. Achiba, H. Kataura, Nature Mater. 2007, 6, 135.

[11] A. E. Porter, M. Gass, K. Muller, J. N. Skepper, P. A. Midgley, M. Welland, Nature Nanotech. 2007, 2, 713.

[12] a) A. Chuvilin, E. Bichoutskaia, M. C. Gimenez-Lopez, T. W. Chamberlain, G. A. Rance, N. Kuganathan, J. Biskupek, U. Kaiser, A. N. Khlobystov, Nature Mater. 2011, 10, 687; b) A. N. Khlobystov, ACS Nano 2013, 5, 9306.

[13] a) A. M. Guloy, Z. Tang, P. B. Miranda, V. I. Srdanov, Adv. Mater. 2001, 13, 833; b) L.-M. Wu, X.-T. Wu, L. Chen, Coord. Chem. Rev. 2009, 253, 2787.

[14] a) M. M. Lee, J. l. Teuscher, T. Miyasaka, T. N. Murakami, H. J. Snaith, Science 2012, 338, 643; b) J. Jun-Yuan, C. Yi-Fang, L. Mu-Huan, P. Shin-Rung, G. Tzung-Fang, C. Peter, W. Ten-Chin, Adv. Mater. 2013, 25, 3727.

[15] R. Kreizman, S. Y. Hong, J. Sloan, R. Popovitz-Biro, A. Albu-Yaron, G. Tobias, B. Ballesteros, B. G. Davis, M. L. H. Green, R. Tenne, Angew. Chem. Int. Ed. 2009, 48, 1230.

[16] Y. Liu, C. Hu, A. Comotti, M. D. Ward, Science 2011, 333, 436.

[17] G. Tobias, L. Shao, C. Salzmann, Y. Huh, M. L. H. Green, J. Phys. Chem. B 2006, 110, 22318. 
[18] a) X. Fan, E. C. Dickey, P. C. Eklund, K. A. Williams, L. Grigorian, R. Buczko, S. T. Pantelides, S. J. Pennycook, Phys. Rev. Lett. 2000, 84, 4621; b) S. Y. Hong, G. Tobias, B. Ballesteros, F. El Oualid, J. C. Errey, K. J. Doores, A. I. Kirkland, P. D. Nellist, M. L. H. Green, B. G. Davis, J. Am. Chem. Soc. 2007, 129, 10966.

[19] B. Palosz, J. Phys.: Cond. Matter 1990, 2, 5285.

[20] a) A. Rubio, J. L. Corkill, M. L. Cohen, Phys. Rev. B 1994, 49, 5081; b) X. Blase, A. Rubio, S. G. Louie, M. L. Cohen, Phys. Rev. B 1995, 51, 6868; c) R. Arenal, O. Stéphan, M. Kociak, D. Taverna, A. Loiseau, C. Colliex, Phys. Rev. Let. 2005, 95, 127601; d) R. Arenal, X. Blase, A. Loiseau, Adv. Phys. 2010, 59, 101.

[21] E. Flahaut, J. Sloan, S. Friedrichs, A. I. Kirkland, K. S. Coleman, Chem. Mater. 2006, 18, 2059.

[22] R. Kreizman, A. N. Enyashin, F. L. Deepak, A. Albu-Yaron, R. Popovitz-Biro, G. Seifert, R. Tenne, Adv. Funct. Mater. 2010, 20, 2459.

[23] A. N. Enyashin, R. Kreizman, G. Seifert, J. Phys. Chem. C 2009, 113, 13664.

[24] M. Wilson, Nano Lett. 2004, 4, 299.

[25] V. C. Holmberg, M. G. Panthani, B. A. Korgel, Science 2009, 326, 405.

[26] P. M. F. J. Costa, U. K. Gautam, Y. Bando, D. Golberg, Nature Commun. 2011, 2, 421.

[27] E. Nakamura, Angew. Chem. Int. Ed. 2013, 52, 236.

[28] a) A. N. Khlobystov, K. Porfyrakis, M. Kanai, D. A. Britz, A. Ardavan, H. Shinohara, T. J. S. Dennis, G. A. D. Briggs, Angew. Chem. Int. Ed. 2004, 43, 1386; b) D. Golberg, P. M. F. J. Costa, M. Mitome, S. Hampel, D. Haase, C. Mueller, A. Leonhardt, Y. Bando, Adv. Mater. 2007, 19, 1937; c) P. M. F. J. Costa, D. Golberg, M. Mitome, S. Hampel, A. Leonhardt, B. Buchner, Y. Bando, Nano Lett. 2008, 8, 3120; d) J. Sloan, G. Matthewman, C. Dyer-Smith, A. Y. Sung, Z. Liu, K. Suenaga, A. I. Kirkland, E. Flahaut, ACS Nano 2008, 2, 966; e) P. M. F. J. Costa, U. K. Gautam, Y. Bando, D. Golberg, Carbon 2011, 49, 3747. 
[29] J. L. Hutchison, N. Grobert, R. M. Zakalyukin, A. A. Eliseev, M. V. Chernisheva, A. S. Kumskov, Y. V. Grigoriev, A. V. Krestinin, B. Freitag, N. A. Kiselev, AIP Conference Proceedings 2008, 999, 79.

[30] a) J. Sloan, A. I. Kirkland, J. L. Hutchison, M. L. H. Green, Chem. Commun. 2002, 1319; b) B. Ballesteros, G. Tobias, M. A. H. Ward, M. L. H. Green, J. Phys. Chem. C 2009, 113, 2653; c) E. Fidiani, P. M. F. J. Costa, A. U. B. Wolter, D. Maier, B. Buechner, S. Hampel, J. Phys. Chem. C 2013, 117, 16725.

[31] a) G. Brown, S. R. Bailey, J. Sloan, C. Xu, S. Friedrichs, E. Flahaut, K. S. Coleman, J. L. Hutchison, R. E. Dunin-Borkowski, M. L. H. Green, Chem. Commun. 2001, 845; b) A. Ilie, J. S. Bendall, K. Nagaoka, S. Egger, T. Nakayama, S. Crampin, ACS Nano 2011, 5, 2559.

[32] M. Amato, S. Ossicini, R. Rurali, Nano Lett. 2011, 11, 594.

[33] B. Ballesteros, G. Tobias, L. Shao, E. Pellicer, J. Nogues, E. Mendoza, Small 2008, 4, 1501.

[34] a) J. M. Soler, E. Artacho, J. D. Gale, A. García, J. Junquera, P. Ordejón, D. SánchezPortal, J. Phys.: Cond. Matter 2002, 14, 2745; b) G. Kresse, J. Furthmüller, Comput. Mat. Sci. 1996, 6, 15.

[35] D. M. Ceperley, B. J. Alder, Phys. Rev. Lett. 1980, 45, 566.

[36] S. Grimme, J. Comp. Chem. 2006, 27, 1787. 


\section{a}

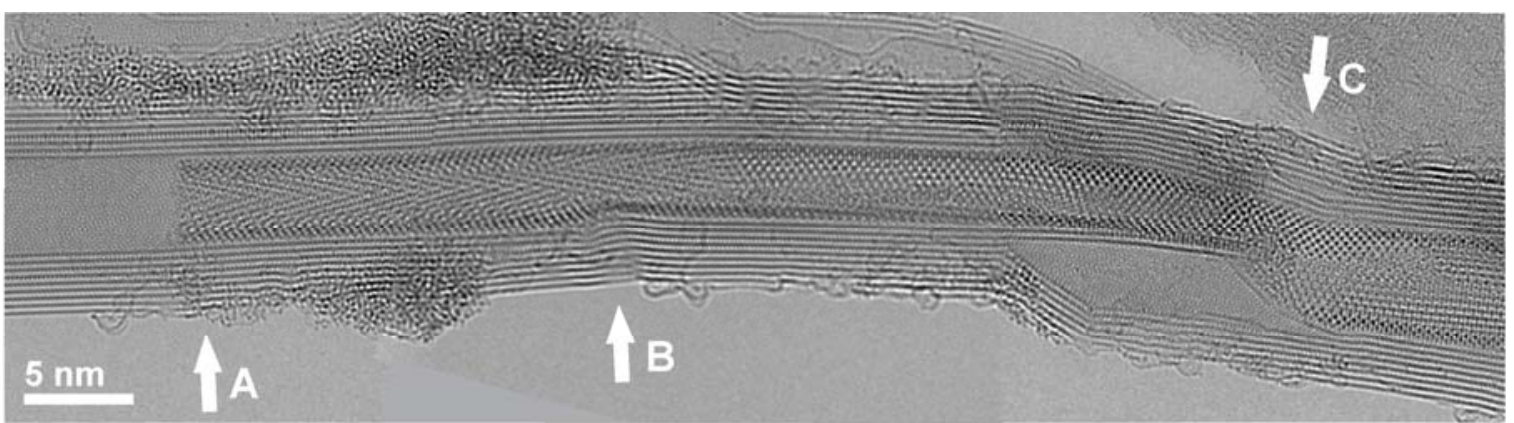

b

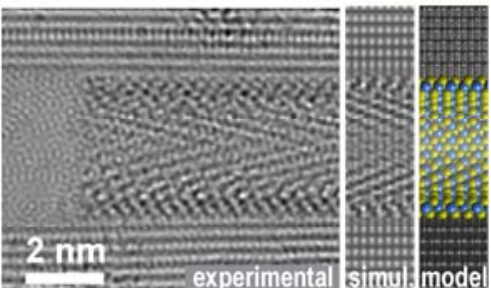

C
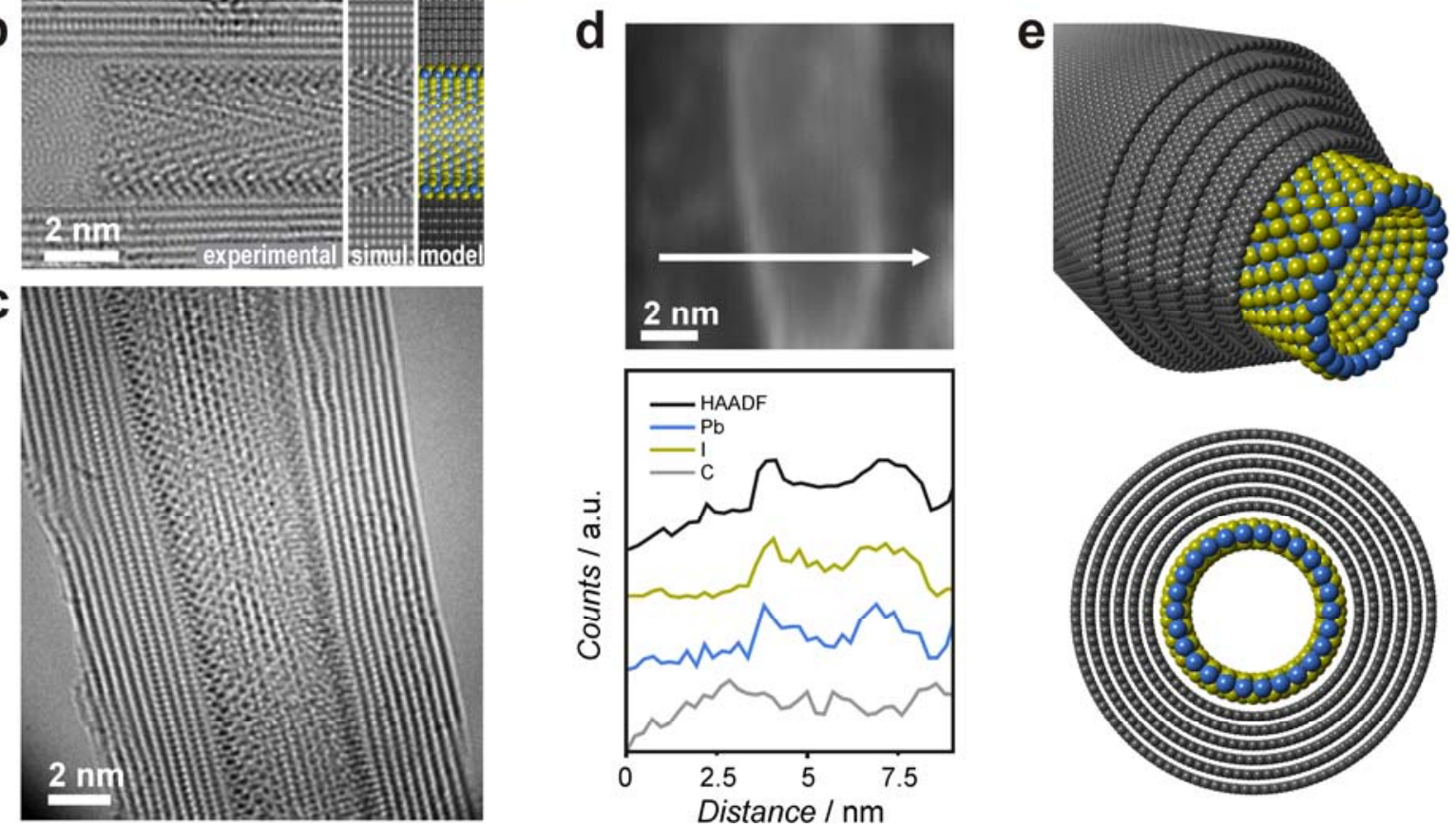

Figure 1. HRTEM and STEM analysis of the synthesised materials. a) Aberration-corrected HRTEM images of a single-layered $\mathrm{PbI}_{2}$ nanotube. b) Detail of the HRTEM image (area A) with its corresponding simulation and model (cross section along the main axis). c)

Aberration-corrected HRTEM image of another single-layered PbI2 nanotube d) EDS-STEM line profiles confirming the presence of a $\mathrm{PbI}_{2}$ nanotube. e) Schematic representation of the grown single-layered $\mathrm{PbI}_{2}$ tubular materials (cyan and green spheres representing $\mathrm{Pb}$ and I atoms, respectively) within the inner cavities of a multiwalled carbon nanotubes (grey spheres). A cross section across the main axis of the tube is also included for clarity. 

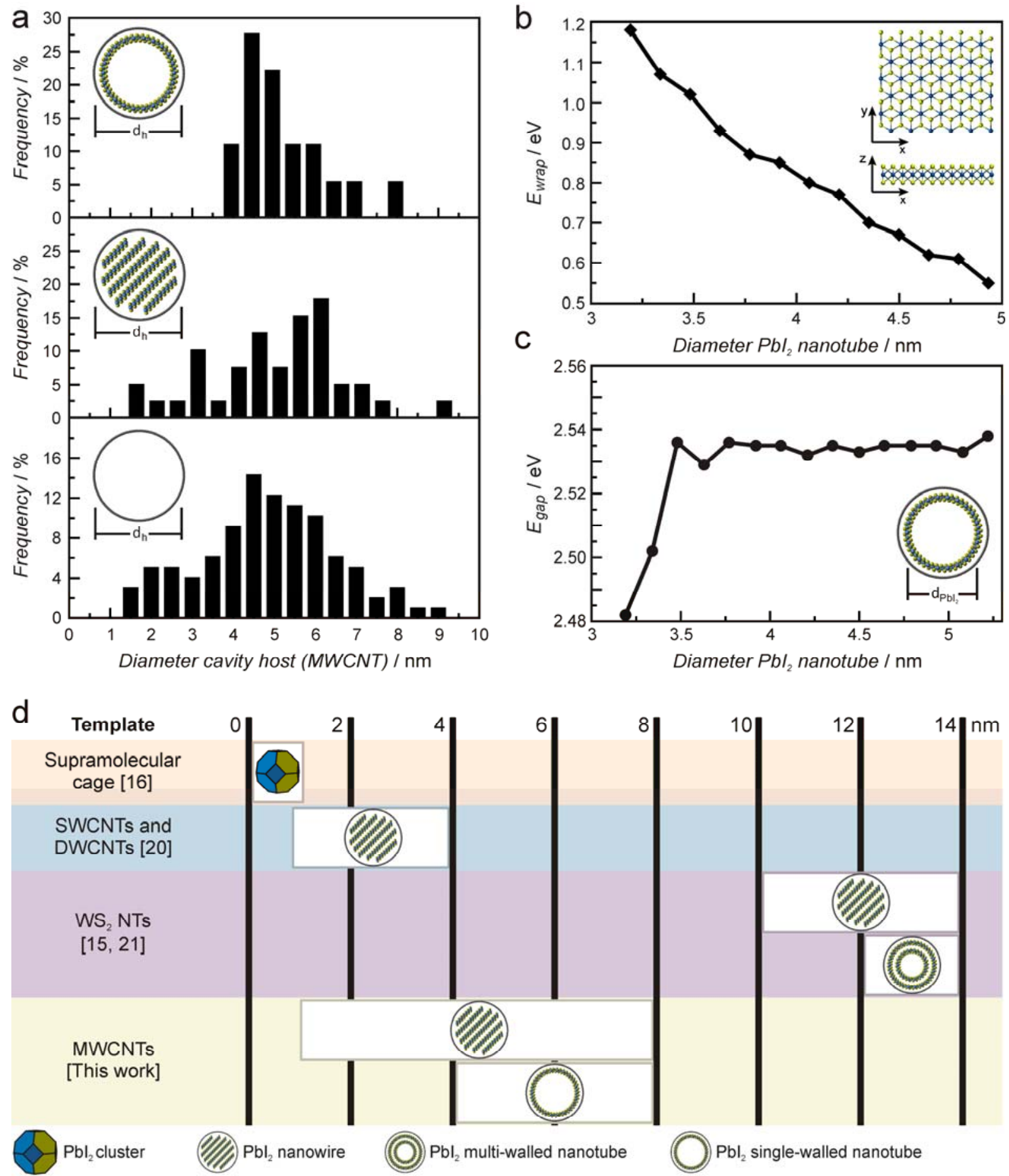

Figure 2. Role of the diameter on the formation, stability and properties of single-layered inorganic nanotubes. a) Histograms of the MWCNT cavity (cavity of the host), when $\mathrm{PbI}_{2}$ nanotubes (top) or $\mathrm{PbI}_{2}$ nanorods (center) are observed; empty MWCNTs (bottom) are also included for comparison. b) Wrapping energy as a function of the $\mathrm{PbI} 2$ nanotube diameter. The wrapping energy is defined as $E_{w r a p}=E_{N T}-E_{M L}$, where $E_{N T}$ is the total energy of the $\mathrm{PbI}_{2}$ nanotube and $\mathrm{E}_{\mathrm{ML}}$ is the total energy of a flat stand-alone $\mathrm{PbI}_{2}$ monolayer of the same size, i.e. 
the unrolled nanotube. The wrapping axis is $x$ (inset) and the energy is given for unit cell of the nanotube. Note that the $\mathrm{x}, \mathrm{y}, \mathrm{z}$ axis correspond to the fragment of $\mathrm{PbI} 2$ layer used to model the nanotubes and not to the bulk $\mathrm{PbI}_{2}$ structure. c) Band-gap vs diameter of the $\mathrm{PbI}_{2}$ singlelayered nanotube. d) Summary on the formation of $\mathrm{PbI}_{2}$ nanostructures using templateassisted growth (from this and previous works) with respect to the diameter of the hosts.
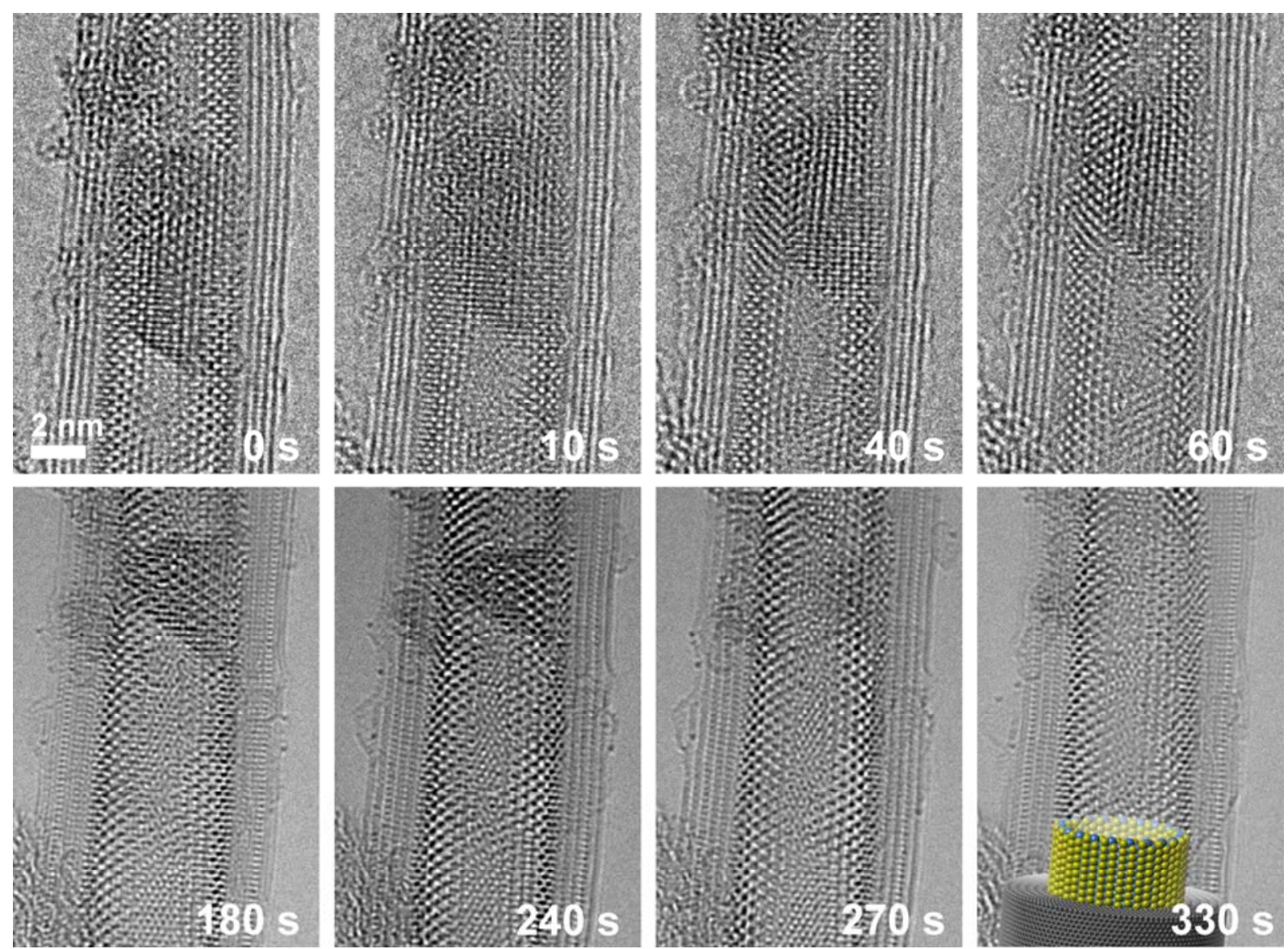

Figure 3. Dynamics under the electron beam radiation. Sequence of images showing the transformation from a nanorod fragment onto a single-layered nanotubes using an electron dose of $\sim 0.610^{4} \mathrm{e}^{-} \AA^{-2}$. A schematic representation is included in the last image; cyan, green and grey spheres representing $\mathrm{Pb}$, I and $\mathrm{C}$ atoms, respectively. 

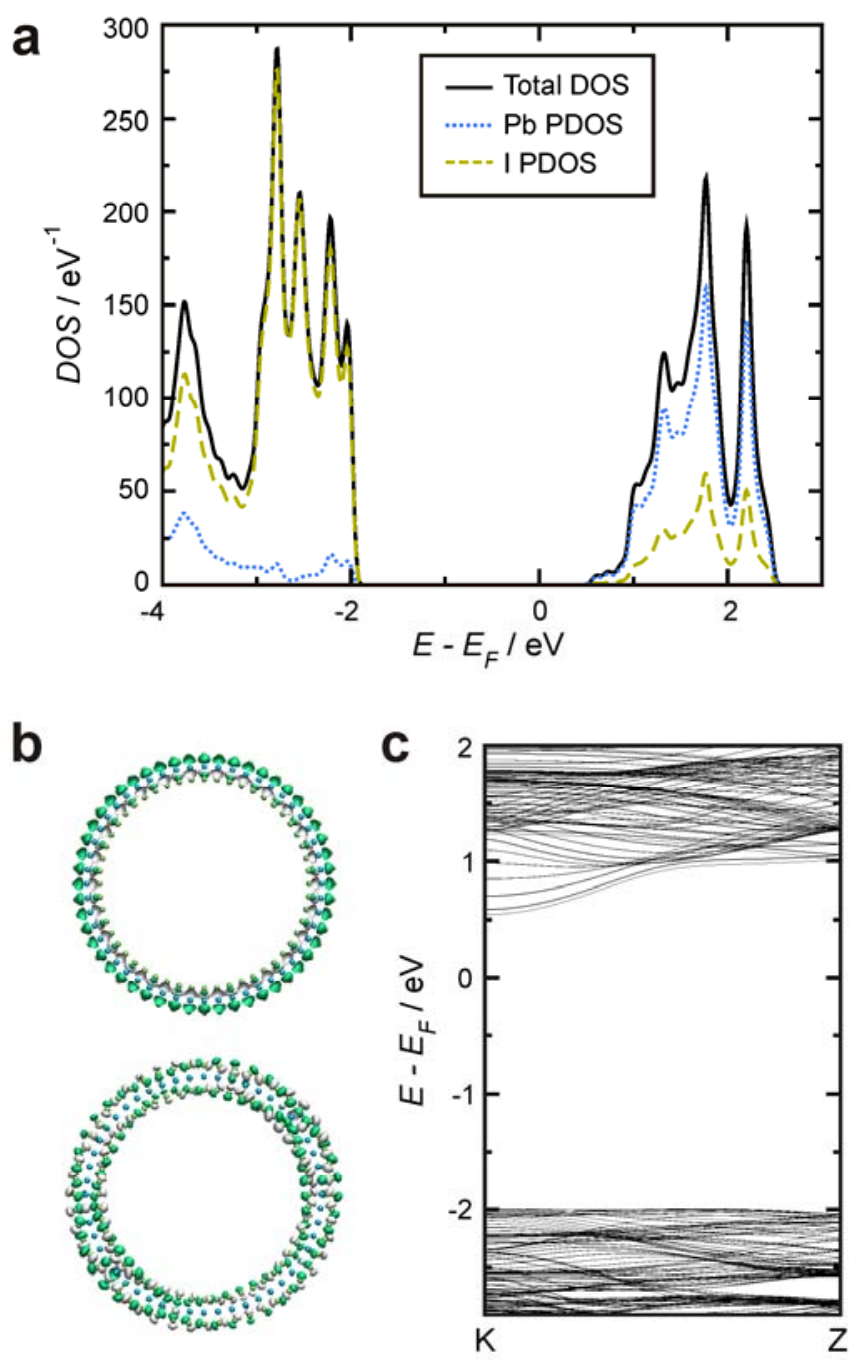

Figure 4. Electronic structure of single-layered $\mathrm{PbI}_{2}$ nanotubes. a) Total density of states and projections on $\mathrm{Pb}$ and I atoms. b) The wave-function of HOMO (bottom) and LUMO (top) shows the different localization: on I atoms the former, on $\mathrm{Pb}$ atoms the latter. c) Band structure diagram. The data presented correspond to a nanotube of $3.77 \mathrm{~nm}$ in diameter. 


\section{Supporting Information}

Synthesis of $\mathrm{PbI}_{2}$ Single-Layered Inorganic Nanotubes Encapsulated Within Carbon Nanotubes

Laura Cabana, Belén Ballesteros, Eudar Batista, César Magén, Raúl Arenal, Judith Oro-Solé, Riccardo Rurali, and Gerard Tobias*
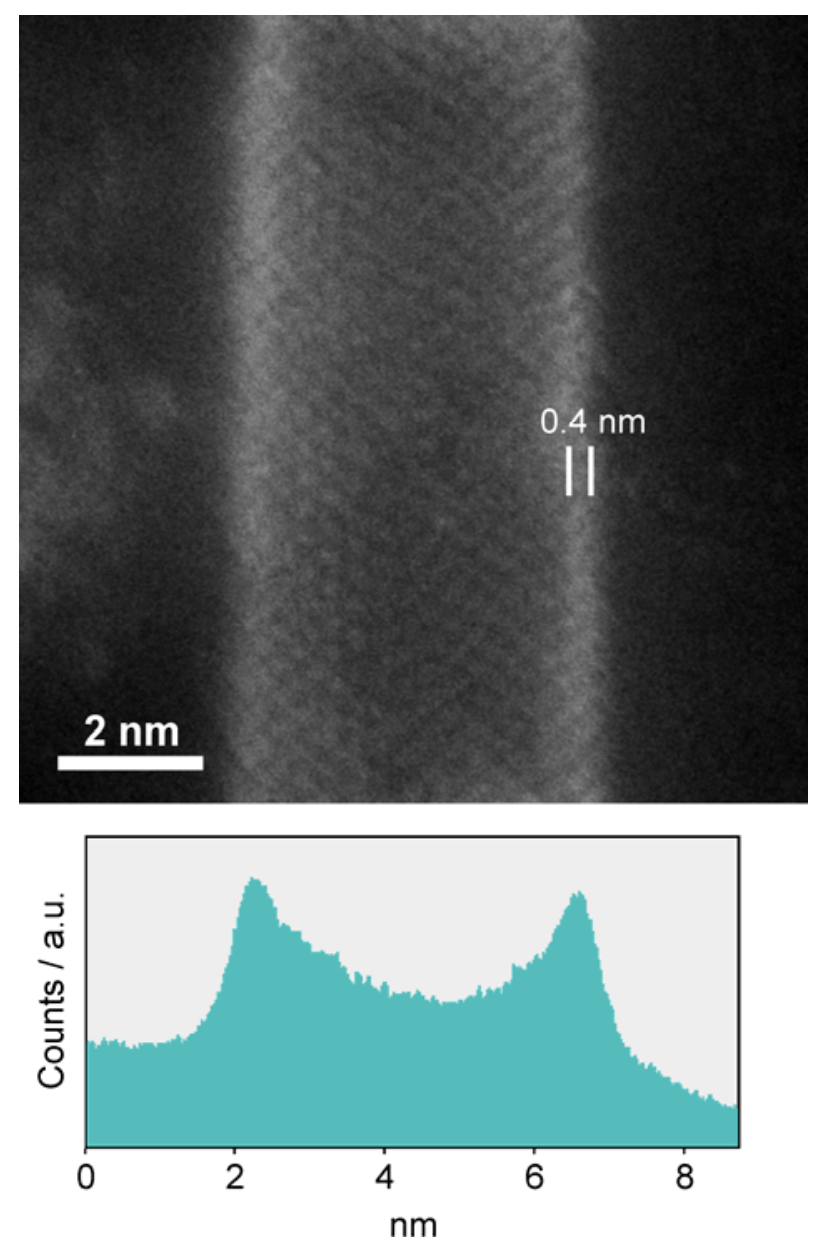

Figure S1. STEM study of a single-layered $\mathbf{P b I}_{2}$ nanotube. HAADF HRSTEM image with its corresponding intensity line profile (integrated along 25 pixels). 
a

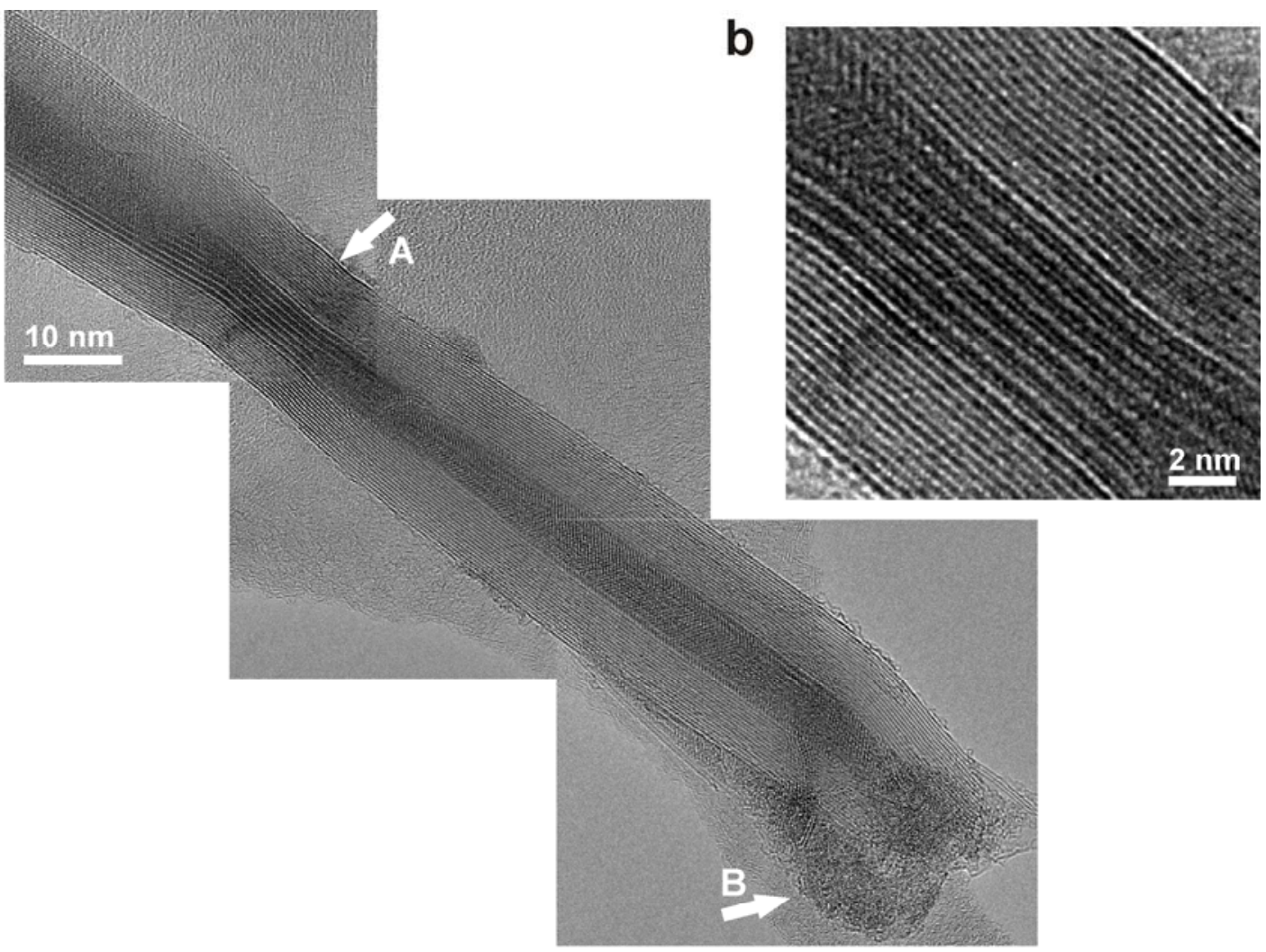

c
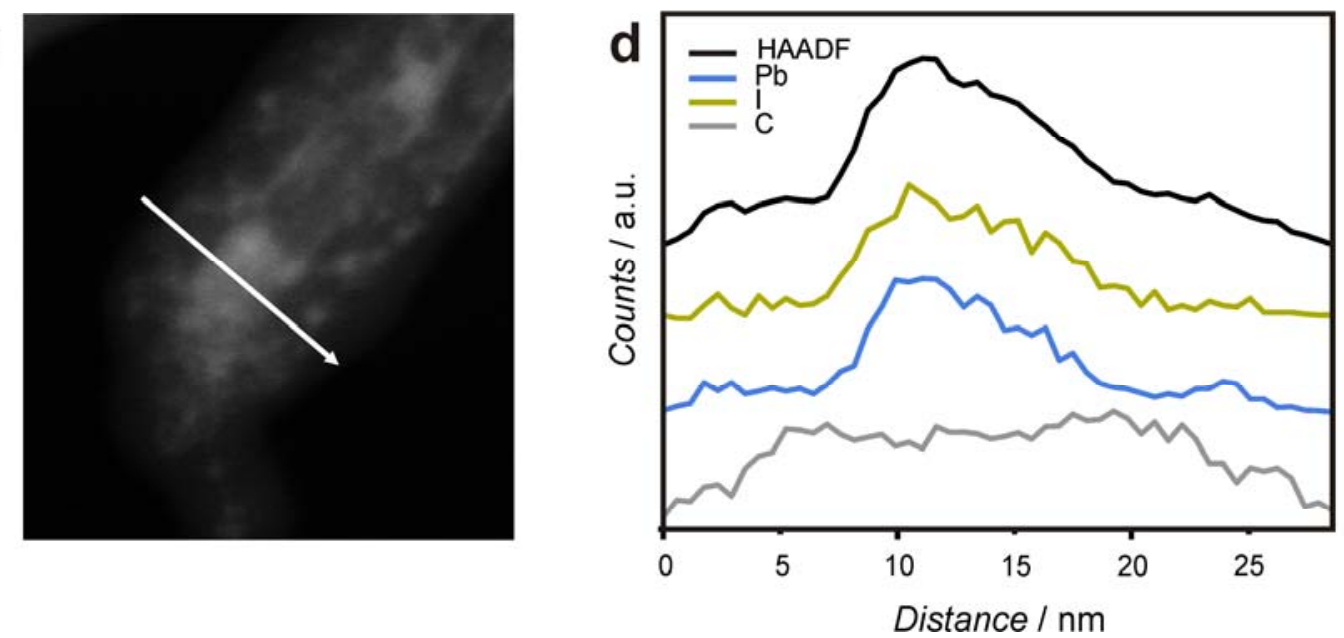

Figure S2. HRTEM and STEM analysis of a $\mathbf{P b I}_{2}$ nanorod. a) and b) Aberration-corrected HRTEM images. A higher magnification of area $A$ is presented in $b$ ) where the layered structure of $\mathrm{PbI}_{2}$ can be better appreciated. A $\mathrm{PbI}_{2}$ reservoir can be seen in area $B$ filling the tip of the CNT. c) HAADF STEM image and d) EDS chemical profile of a PbI2 nanorod confined within a multiwalled carbon nanotube. 

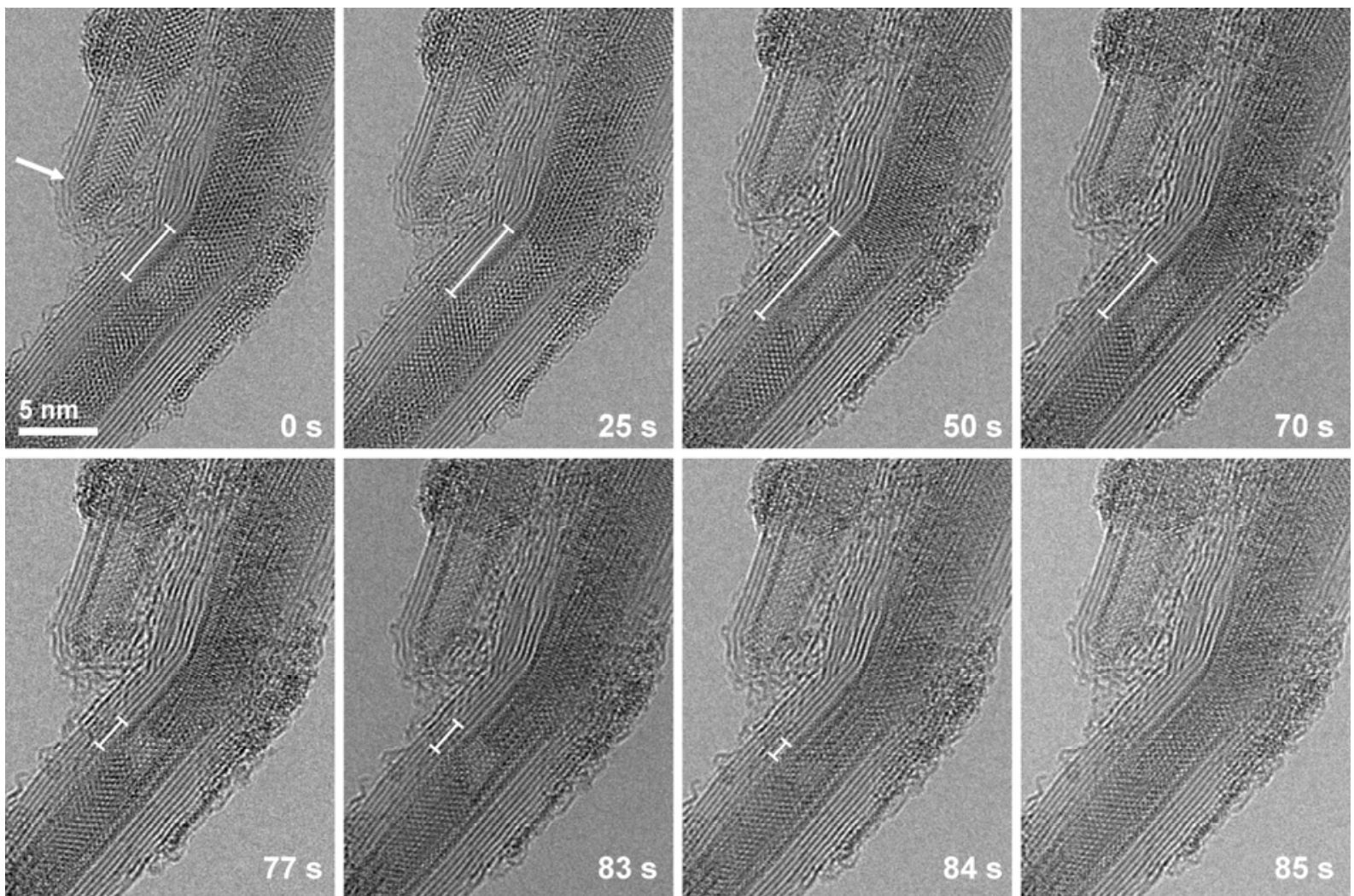

Figure S3. Dynamics under the electron beam radiation. Sequence of images showing the transformation from a $\mathrm{PbI}_{2}$ nanotube fragment onto a nanorod using an electron dose of $\sim 0.2$ $10^{4} \mathrm{e}^{-} \AA^{-2}$. For clarity a white bar has been drawn along the length of the nanotubular fragment, which initially expands up to $50 \mathrm{~s}$ and then starts to contract until its complete disappearance. The arrow points to the tip of a single-layered $\mathrm{PbI}_{2}$ nanotube that is present into an adjacent CNT. 

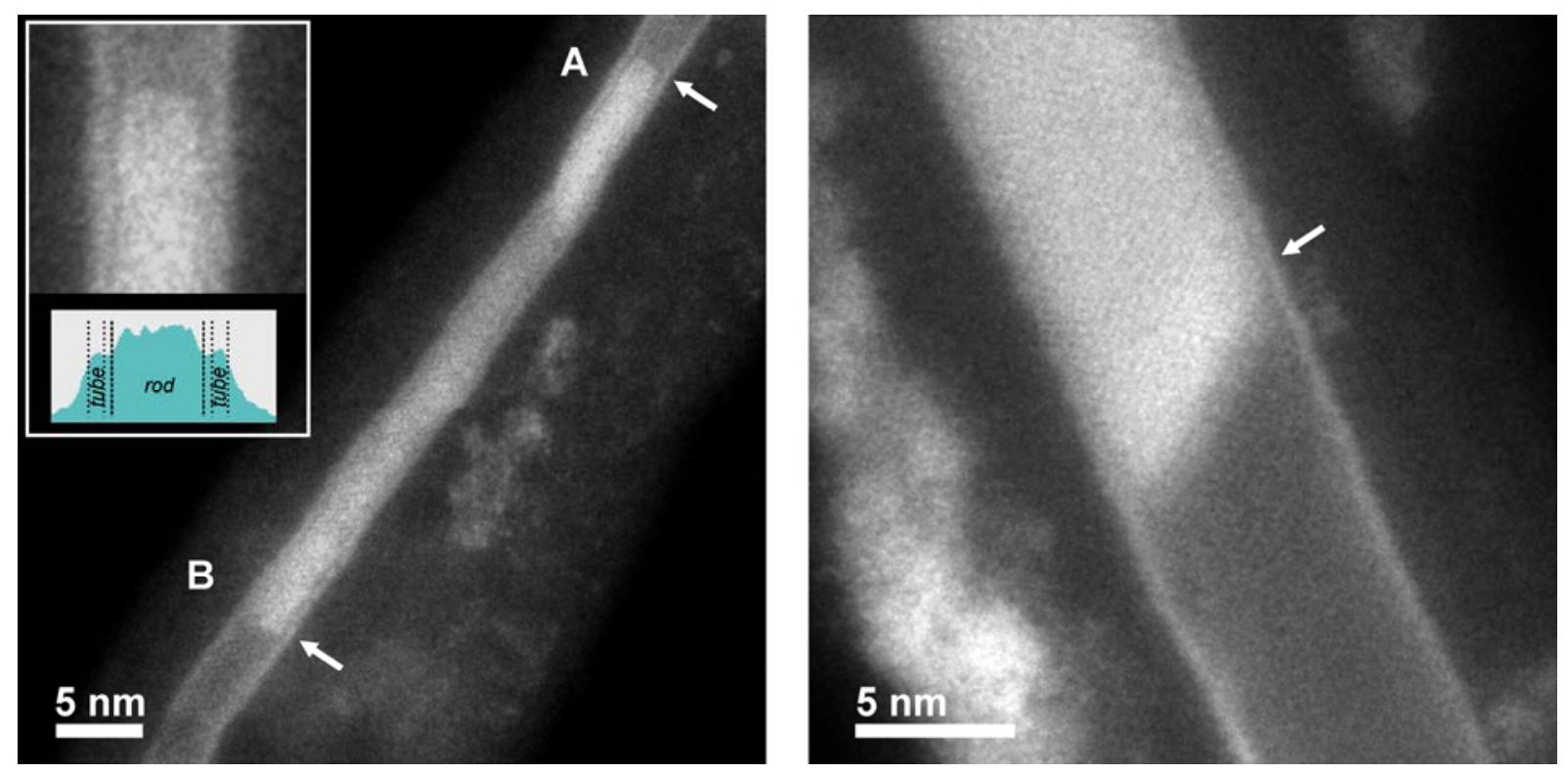

Figure S4. HAADF STEM images of nanotube nanorod junctions. In both images a $\mathrm{PbI}_{2}$ nanorod appears to be confined within a $\mathrm{PbI}_{2}$ nanotube. The arrows point at the end of the nanorods. The inset shows a higher magnification image of area A with its corresponding line intensity profile (integrated along 25 pixels). 